\title{
Epidemiology and Risk Factors for Development of Diabetic Retinopathy
}

\author{
Amira Kashwa', Amr Abdelkader ${ }^{2}$, Hossam Abouelkheir ${ }^{2}$, Hamza Ahmad ${ }^{2}$ \\ 1: $\mathrm{MBBCH}$, Mansoura University \\ 2: Ophthalmology Department, Faculty of Medicine, Mansoura University, Egypt.
}

Corresponding author: Dr. Amira Ahmad Kashwa, MBBCH, Mansoura University. Egypt. Tel: 01001073304 ; Email: amirakashwa@gmail.com

Received: 18-5-2021, Accepted: 30-8-2021, Published online:16-9-2021

EJO(MOC) 2021;3:128-137.

Running title: Risk factors of diabetic retinopathy.

\begin{abstract}
Background: Diabetic retinopathy (DR) is one of the leading causes of blindness worldwide and patients with this sight-threatening disease are expected to grow as dietary habits are changing especially in developing nations. For the past two decades, optical coherence tomography (OCT) was used as a routine technique for ocular imaging. It is considered as an ideal technique because it is a non-invasive technique and in the same time is able to give high-resolution $(1-10 \mu \mathrm{m})$, depth-resolved, and cross-sectional images.

Objective: Study the epidemiology and the risk factors of diabetic retinopathy among diabetic retinopathy patients attending the outpatient clinic of Mansoura ophthalmic center through one year.

Patients and methods: This cross-sectional descriptive study included 400 eyes of 200 patients with diabetic retinopathy recruited from Mansoura ophthalmic center, Mansoura University Hospitals, Egypt. After obtaining a written informed consent, the cases were subjected to full history taking and full ophthalmological examination. Spectral domain OCT 2000 was used to obtain retinal images. Laboratory investigations were conducted for all the cases included HBA1C, lipid profile and serum creatinine
\end{abstract}

Results: The mean macular thickness was statistically significantly higher in the cases with HTN and cases with hepatitis. There was no statistically significant difference in the macular thickness according to sex, smoking or family history of DM. There was a statistically significant difference in the mean macular thickness between the eyes according to the findings of fundus examination. The highest macular thickness was reported with the eyes with stage 3 non-proliferative diabetic retinopathy (NPDR). Linear regression analysis, cholesterol level and best corrected visual acuity (BCVA) had statistically significant predictive ability for macular thickness

Conclusion: Retinopathy is a common complication of diabetes in diabetic patients. DR at the initial stage of diabetes may prevent disability from blindness caused by DR.

Key words: Diabetes, Diabetic retinopathy, Optical coherence tomography, Macular thickness.

\section{Introduction:}

Diabetes mellitus (DM) is a major medical problem throughout the world. Diabetes causes an array of long-term systemic complications that have considerable impact on the patient, family, and society, as the disease typically affects individuals in their most productive years ${ }^{[1]}$.
The prevalence of diabetes is increasing throughout the world, and this increase appears to be greater in developing countries mostly due to modernization of life and unhealthy dietary patterns ${ }^{[2]}$.

Patients with diabetes often develop ophthalmic complications, such as corneal abnormalities, glaucoma, iris revascularization, cataract, and neuropathies. The most

\footnotetext{
Egyptian Journal of Ophthalmology, a publication of Mansoura Ophthalmic Center.

Address: Mansoura Ophthalmic Center, Mansoura University, Mansoura, Egypt.

Tel. 0020502202064. Fax. 0020502202060.

E-mail: ejo_moc@yahoo.com; tharwatmokbel@yahoo.com
} 
common and potentially most blinding of these complications, however, is diabetic retinopathy (DR) ${ }^{[3]}$.

The progression of retinopathy is gradual, advancing from mild abnormalities, characterized by increased vascular permeability, to moderate, severe non-proliferative till proliferative DR characterized by the growth of new blood vessels on the retina and on the posterior surface of the vitreous $^{[4]}$.

Several factors have been identified as determinants for the development of DR and its progression, including the type of diabetes, family history, duration of DM, age, sex, glycemic control, hypertension, Body mass index , smoking, serum lipids, and presence of microalbuminuria ${ }^{[5-8]}$.

Traditionally, diagnosis of DR has been clinical with adjunctive testing, such as fluorescein angiography (FA) and optical coherence tomography (OCT), used to confirm or quantify clinical suspicion of structural complications, such as neovascularization and macular edema. Except for common metrics, such as central macular thickness or macular volume, the interpretation of OCTs in diabetics has been predominantly subjective ${ }^{[9]}$.

Optical coherence tomography (OCT) is an imaging technique similar to ultrasound, but instead of using sound waves it uses light to achieve much higher resolution images (10-100 times better than ultrasound) ${ }^{[10]}$.

To the best of our knowledge, an accurate, automated screening system for DR based on OCT does not exist with lack of studies in this topic.

\section{Patients and methods}

This is a cross sectional, observational non-interventional study that was conducted in the period between September 2019 to September 2020 in Mansoura ophthalmic center, Mansoura University, Egypt.

This study included 400 eyes of 200 patients with diabetic retinopathy from both genders and from different ages. The cases with the following conditions were excluded; Patients with impaired glucose tolerance, eyes with ocular abnormality other than diabetic macular edema such as vitreoretinal disease and epiretinal membrane and presence of media opacities interfering with reliability of OCT imaging (dense cataract, corneal opacity, uveitis).
After approval from the institutional review board of Mansoura Faculty of Medicine and obtaining an informed written consent from the participants, all cases were subjected to complete history taking.

Full detailed ophthalmic examination was done for all the cases including assessment of the visual acuity (VA) using Landolt's VA chart and then transformed for statistical analysis to logarithm of minimal angle of resolution units ( $\mathrm{Log}$ MAR).

slit lamp biomicroscopy (Haag Streit BP 900) (Haag-Streit, Koeniz, Switzerland) was used to assess corneal clarity, anterior chamber depth and regularity, pupil shape, size, regularity and reactivity, state of the lens and complications of diabetes such as (recurrent styes, xanthelasma, accelerated senile cataract, rubeosis iridis).

Posterior segment examination was conducted using indirect ophthalmoscope and slit lamp biomicroscopy with auxillary lenses.

Optical coherence tomography (OCT) using Spectral domain OCT 1000 [Topcon, Inc., Paramus, NJ, USA] was used to measure the central macular thickness and correlate it with different risk factors.

The following laboratory investigations were done for all the cases (HBA1C, lipid profile and serum creatinine,

\section{Results}

The current study included 200 patients with diabetic retinopathy. The mean age of the cases was $55.23 \pm 9.28$ years. Among the cases, there were 72 males (36\%) and 128 females (64\%). The mean duration of affection with DM among the included cases was $14.17 \pm 6.59$ years with 7 months as the least duration and 30 years as the maximum duration. There were 40 cases $(20 \%)$ who received oral antidiabetic as treatment for DM and 160 cases $(80 \%)$ who received Insulin. The study of the associated comorbidities and risk factors revealed that 20 cases $(10 \%)$ were smokers, 142 cases $(71 \%)$ were hypertensive, hepatitis was present in 42 cases (21\%) and dyslipidaemia in 129 cases (64.5\%). Positive family history of DM was present in 129 cases (64.5\%) and glycaemic control was detected in 130 cases $(65 \%)$.

$14 \%$ of the included eyes had history of cataract surgery, $27.5 \%$ had history of intravitreal injection and 20.5\% had history of laser therapy. Data were shown in table (1) 
Table (1): Demographic, medical and ophthalmological history in study cases.

\begin{tabular}{|c|c|c|}
\hline Items & & Cases $(n=200)$ \\
\hline \multirow[t]{2}{*}{ Age (years) } & Mean \pm SD & $55.23 \pm 9.28$ \\
\hline & Median (min-max) & $57(21-76)$ \\
\hline \multicolumn{3}{|l|}{ Sex } \\
\hline Males & & $72(36 \%)$ \\
\hline Females & & $128(64 \%)$ \\
\hline \multirow[t]{2}{*}{ Duration of the disease (years) } & Mean $\pm \mathrm{SD}$ & $14.17 \pm 6.59$ \\
\hline & Median (min-max) & 15 years ( 7 months-30 years) \\
\hline \multicolumn{3}{|l|}{ Treatment } \\
\hline Oral antidiabetic & & $40(20 \%)$ \\
\hline Insulin & & $160(80 \%)$ \\
\hline \multicolumn{3}{|l|}{ Medical history } \\
\hline Smoking & & $20(10 \%)$ \\
\hline Hypertension & & $142(71 \%)$ \\
\hline Hepatitis & & $42(21 \%)$ \\
\hline Dyslipidemia & & $129(64.5 \%)$ \\
\hline Positive Family history & & $129(64.5 \%)$ \\
\hline \multicolumn{3}{|l|}{ Glycemic control } \\
\hline Controlled (HBA1C $\leq 7.5)$ & & $130(65 \%)$ \\
\hline Uncontrolled (HBA1C > 7.5) & & $70(35 \%)$ \\
\hline \multicolumn{3}{|l|}{ Ophthalmological history } \\
\hline History of cataract surgery & & $56(14 \%)$ \\
\hline History of IVI & & $110(27.5 \%)$ \\
\hline History of LASER therapy & & $82(20.5 \%)$ \\
\hline
\end{tabular}

Fundus examination of the included cases revealed that mild NPDR was found in $30.25 \%$ of the eyes, moderate NPDR

There was no statistically significant difference in the mean macular thickness between the male and female cases $(p=0.112)$. Also, there was no statistically significant difference in the mean macular thickness between smokers and non-smokers $(\mathrm{p}=0.236)$, or between cases with positive and negative family history $(\mathrm{p}=0.661)$. The mean macular thickness was statistically significant higher in the cases with was detected in $42.75 \%$ of the cases, while sever NPDR was found in $16 \%$ and PDR in $11 \%$ of the eyes.

hypertension and cases with hepatitis $(\mathrm{p}=0.031$ and 0.015$)$ respectively.

Also, there was a statistically significant difference in the mean macular thickness between the eyes according to the findings of fundus examination. The highest macular thickness was reported with the eyes with sever NPDR. Data were presented in table (2). 
Table (2): Effect of the risk factors on macular thickness

\begin{tabular}{|c|c|c|c|}
\hline Variables & no & Thickness & p-value \\
\hline Sex & & & 0.112 \\
\hline Males & 72 & $314.89 \pm 166.15$ & \\
\hline Females & 128 & $275.16 \pm 123.01$ & \\
\hline Smoking & & & 0.236 \\
\hline Yes & 20 & $326.16 \pm 158.11$ & \\
\hline No & 180 & $286.15 \pm 139.25$ & \\
\hline HTN & & & $0.031 *$ \\
\hline Yes & 142 & $300.84 \pm 144.87$ & \\
\hline No & 58 & $265.84 \pm 136.04$ & \\
\hline Hepatitis & & & $0.015^{*}$ \\
\hline Yes & 42 & $335.18 \pm 157.34$ & \\
\hline No & 158 & $277.34 \pm 135.2$ & \\
\hline Family history & & & 0.661 \\
\hline Positive & 129 & $289.54 \pm 137.41$ & \\
\hline Negative & 71 & $290.26 \pm 141.34$ & \\
\hline \multicolumn{4}{|l|}{ Fundus examination } \\
\hline Mild NPDR & $121(30.25 \%)$ & $211.89 \pm 63.86$ & \\
\hline Moderate NPDR & $171(42.75 \%)$ & $305.7 \pm 139.1$ & $<0.001^{*}$ \\
\hline Sever NPDR & $64(16 \%)$ & $405.45 \pm 187.51$ & \\
\hline PDR & $44(11 \%)$ & $286.04 \pm 102.4$ & \\
\hline
\end{tabular}

There was a statistically significant positive triglycerides level. There was a statistically significant correlation between macular thickness with age, disease negative correlation between macular-thickness and BCVA. duration, HBA1C, serum creatinine, cholesterol level and Data was presented in table (3).

Table (3): Correlation between macular thickness and other parameters in the study

\begin{tabular}{ccc}
\hline \multirow{2}{*}{ Age } & & Macular thickness \\
\hline \multirow{2}{*}{ Disease duration } & $\mathrm{r}$ & 0.195 \\
& $\mathrm{p}$ & $0.006^{*}$ \\
HBA1C & $\mathrm{r}$ & 0.172 \\
Creatinine & $\mathrm{p}$ & $0.015^{*}$ \\
& $\mathrm{r}$ & 0.388 \\
Cholesterol & $\mathrm{p}$ & $<0.001^{*}$ \\
\multirow{2}{*}{ Triglycerides } & $\mathrm{r}$ & 0.149 \\
& $\mathrm{p}$ & $0.036^{*}$ \\
BCVA & $\mathrm{r}$ & 0.582 \\
& $\mathrm{p}$ & $<0.001^{*}$ \\
& $\mathrm{r}$ & 0.469 \\
& $\mathrm{p}$ & $<0.001^{*}$ \\
& $\mathrm{r}$ & -0.495 \\
& $\mathrm{p}$ & $<0.001^{*}$ \\
\hline
\end{tabular}

R: Spearman's correlation

P: probability

*: Statistically significant $(\mathrm{p}<0.05)$

Cholesterol level and BCVA had statistically significant predictive ability for macular thickness by linear regression analysis, as shown in table (4). 
Table (4): Linear regression analysis for macular thickness in the cases of the study

\begin{tabular}{|c|c|c|c|c|c|c|c|}
\hline & \multicolumn{2}{|c|}{$\begin{array}{l}\text { Unstandardized } \\
\text { Coefficients }\end{array}$} & \multirow{3}{*}{$\begin{array}{c}\text { Standardized } \\
\text { Coefficients } \\
\text { Beta }\end{array}$} & \multirow[t]{3}{*}{$\mathrm{t}$} & \multirow[t]{3}{*}{ Sig. } & \multicolumn{2}{|c|}{$\begin{array}{l}\text { 95.0\% Confidence Interval } \\
\text { for B }\end{array}$} \\
\hline & B & Std. Error & & & & Lower & Upper \\
\hline & & & & & & Bound & Bound \\
\hline (Constant) & 122.267 & 77.823 & & 1.571 & 0.118 & -31.257 & 275.790 \\
\hline Age & -0.491 & 0.994 & -0.033 & -0.494 & 0.622 & -2.451 & 1.469 \\
\hline HBA1C & 1.496 & 5.479 & 0.019 & 0.273 & 0.785 & -9.313 & 12.305 \\
\hline Creatinine & -30.649 & 42.124 & -0.048 & -0.728 & 0.468 & -113.748 & 52.450 \\
\hline cholesterol & 1.117 & 0.175 & 0.554 & 6.377 & $<0.001 *$ & 0.771 & 1.462 \\
\hline Triglycerides & -0.111 & 0.214 & -0.043 & -0.516 & 0.606 & -0.533 & 0.312 \\
\hline BCVA & -180.583 & 67.319 & -0.278 & -2.683 & 0.008* & -313.385 & -47.782 \\
\hline
\end{tabular}

\section{Discussion:}

The current study addressed the epidemiology and the risk factors of diabetic retinopathy among diabetic retinopathy patients attending the outpatient clinic at Mansoura ophthalmic center through one year. This study included 400 eyes of 200 diabetic patients with different stages of diabetic retinopathy.

Similar results were reported by Abdeen et al. (2018) who included 500 eyes of 287 patients with different stages of diabetic retinopathy, 115 males (40.07\%) and 172 females (59.93\%). Their ages ranged from 20 to 80 years, with mean age 55.98 and standard deviation $\pm 9.81^{[11]}$.

Females constituted nearly about two thirds (64\%) of our diabetic population, which is consistent with previous epidemiological studies carried out in Egypt ${ }^{[12,13]}$.

On the other hand, gender was not found to be an important risk factor for developing DR in most other studies ${ }^{[13-16]}$. However, other studies found males to be more prone to develop DR than females, as in UAE ${ }^{[6]}$ and Oman ${ }^{[17]}$.

There has been considerable inconsistency of the prevalence of DR from one study to another even within the same country ${ }^{[6,17-21]}$. The reason is partly explained by the different methodology used and the different population sample investigated in each study design

In the current study, the mean duration of affection with DM among the included cases was $14.17 \pm 6.59$ years with 7 months as the least duration and 30 years as the maximum duration. Similar duration was also reported by Tang et al.
(2017) who showed that the duration of DM among the included subjects was $13.5 \pm 9.37$ years ${ }^{[5]}$.

This was shorter than the duration reported in a study conducted in Saudi Arabia by Ahmed and his colleagues where the mean age of onset and mean duration of diabetes were 43.91 and 13.4 years, respectively. The difference could be explained by different criteria of the included subjects.

Regarding the results of fundus examination in the included cases, mild NPDR was found in $30.25 \%$ of the eyes, moderate NPDR in $42.75 \%$ of the cases, sever NPDR in $16 \%$ and PDR in $11 \%$ of the eyes.

Similar to our results, the study conducted by Abdeen et al. (2018) showed that mild / moderate NPDR was detected in $11.6 \%$ of the eyes, sever NPDR in $31 \%$, mild / moderate PDR in $28.6 \%$, Sever PDR in $22.2 \%$ of the cases and advanced diabetic eye disease in $6.6 \%$ of the cases ${ }^{[11]}$.

Furthermore, Tang et al. (2017) showed that among 434 eyes from 286 patients with diabetes included in their study, there were 171 eyes with no DR, 120 eyes with mild NPDR, 114 eyes with moderate NPDR, 25 eyes with severe NPDR and 4 eyes with PDR ${ }^{[5]}$.

Our results also agreed with Ahmed et al. (2016) who showed that the overall prevalence of DR was $146(36.4 \%)$. Mild NPDR was found in $57.5 \%$ of the patients, moderate NPDR in $19.9 \%$ and severe NPDR in $11.0 \%$ while $11.6 \%$ of diabetic patients had PDR ${ }^{[8]}$. 
The present study showed a higher prevalence of PDR (11\%) than that of $\operatorname{KSA}(6.4 \%)^{[22]}$, but lower than that reported from Oman $(12.8 \%)^{[6,17]}$.

Our study found no significant gender difference in the development of DR, which is in accord with multiple studies mostly from the middle east and Saudi Arabia ${ }^{[17,23-26]}$, but it is in contrast to a study from Sweden ${ }^{[27]}$, which documents higher rates for women than men ${ }^{[22]}$; and studies from KSA, India, and UAE where DR was observed to be more prevalent in male diabetics ${ }^{[\mathbf{6}, 28]}$.

In the current study, there was a statistically significant difference in the mean age between the cases according to the findings of fundus examination. There was an increase in the mean age with the increase of the stage of DR. Also, there was a statistically significant positive correlation between macular thickness with age.

This disagreed with Macky et al. who reported that the risk between increasing age and developing DR was only applied to patients $>30$ years in their study ${ }^{[12]}$.

This might be explained as their study had higher incidence of type 1 diabetes that is more manifested in younger patients, which could be a stronger influential factor than age and duration of DM

The duration of diabetes could be considered as the single most important and consistent risk factor for the development of DR. In the current study, there was a statistically significant difference in the mean disease duration between the cases according to the findings of fundus examination. There was a reported increase in the disease duration with the increase of the stage of DR with the longest duration was reported with the eyes with sever NPDR. Also, there was a statistically significant positive correlation between macular thickness with disease duration.

This came in accordance with Ahmed et al. (2016) who showed a significant difference in mean duration among the four stages of retinopathy; $(P=0.001)$. Post-hoc test revealed that the duration of DM was significantly different for PDR $(P=0.003)$ in comparison to NPDR. Similarly, the total mean cholesterol was different among the four stages $(P=0.019)^{[8]}$.

This agreed with the Egyptian study conducted by Macky et al. who showed that there was a statistically significant increase in the prevalence of DR with longer durations of diabetes from one group to another $(p<0.001)^{[12]}$.

The results of our study indicate that retinopathy increases with younger age at onset of diabetes and showed a significant association between DR and duration of diabetes, which is consistent with most of the previous studies ${ }^{[14,22,29,30]}$. A study from Sweden documents that prevalence of DR reached $100 \%$ after 30 years of diabetes ${ }^{[27]}$

In the current study, there was a statistically significant difference in the mean HBA1C between the cases according to the findings of fundus examination. There was an increase in the level of HBA1C with the increase of the stage of DR with the highest HBA1C level was reported with the eyes with sever NPDR. Also, there was a statistically significant positive correlation between macular thickness with HBA1C.

The results of our study were similar to the prevalence studies, cohort studies have identified systemic parameters of quality of metabolic control (i.e., hyperglycaemia or the HbA1c level) to be consistently associated with incidence and progression of DR ${ }^{[31-35]}$.

On the contrary, Macky and his colleagues did not find any significant difference in the level of HBA1C with the different stages of DR. This surprising finding was also reported previously for diabetic patients in the UAE ${ }^{[19]}$.

Also, our results disagreed with a longitudinal study by Aiello et al. reported that the prevalence of DR in longstanding diabetes is not dependent on the control of the disease.

However, this could be due to the fact that since HBA1c reflects only the previous 3 months of control, it does not say anything about patients' glycemic control over the years.

In the current study, there was a statistically significant difference in the mean BCVA between the cases according to the findings of fundus examination. There was decrease in the BCVA with the increase of the stage of DR with the lowest BCVA was reported with the eyes with sever NPDR. Also, there was a statistically significant negative correlation between macular thickness with BCVA.

This agreed with the results of the Diabetic Retinopathy Clinical Research Network study conducted by Browning and his colleagues who showed that by analysis of patients in DR treated with laser showed a linear relationship between central 
retinal thickness and visual acuity, but there was substantial variation in visual acuities at any given retinal thickness. Many eyes with thickened maculae had excellent visual acuity, and many eyes with maculae of normal thickness had decreased visual acuity. Suggesting macular thickness is just one of several variables affecting visual acuity ${ }^{[36]}$.

In the current study, there was a statistically significant positive correlation between macular thickness with serum creatinine levels.

Similar observations on chronic kidney diseases as factor associated with DR were made in the Korea National Health and Nutrition Examination Survey 2008-2010 [37]. Also, in the Beijing Eye Study, a higher 10-year incidence of DR was significantly associated with higher serum concentration of creatinine $(\mathrm{p}=0.02$; OR $1.01 ; 95 \%$ CI $1.002-1.022)$ in a multivariate analysis ${ }^{[38]}$.

In the current study, there was a statistically significant positive correlation between macular thickness with cholesterol level and triglycerides level. Moreover, by linear regression analysis, cholesterol level had statistically significant predictive ability for macular thickness.

Our results came in agreement with Ahmed et al. (2016) who showed that the total cholesterol level was significantly higher in the severe grade of DR, which may indicate that it could be a risk of the progression of DR to severe retinopathy, a finding that emphasizes the importance of good lipid control as a preventive measure for the progression of retinopathy ${ }^{[8]}$.

In a meta-analysis of case-control studies, the mean concentrations of total serum cholesterol (mean difference [mg/dL]: 30.1; 95\% CI 21.1-39.0; p < 0.001), low-density lipoproteins (18.6; 95\% CI 5.8-31.4; $\mathrm{p}<0.05)$, and serum triglycerides (24.8; 95\% CI 9.2-40.4; p < 0.05) were significantly higher in patients with DME than those in individuals without macular oedema ${ }^{[39]}$.

On the other hand, population-based cross-sectional studies did not find any significant associations between dyslipidemia with DR ${ }^{[40,41]}$.

Interestingly, fenofibrate, which reduces the levels of lowdensity lipoprotein, very low-density lipoprotein and triglyceride and increases high-density lipoprotein levels, as compared to placebo has been reported to reduce the risk of requiring laser treatment for proliferative DR or DME by $31 \%{ }^{[42]}$.

The difference between the studies could be explained by different sample size and different characteristic of the included cases.

In the current study, 142 cases (71\%) were hypertensive. The mean macular thickness was statistically significant higher in the cases with HTN as compared with nonhypertensive cases $(\mathrm{p}=0.031)$.

This agreed with Macky et al. (2011) who showed that 61\% of diabetics in their study population were found to be hypertensive of whom $77 \%$ were controlled. Diabetic patients with hypertension had about twice the risk of developing DR than non-hypertensive diabetics ( 25 vs. 13\%). Also, diabetics with uncontrolled hypertension had about 3 times the risk of developing DR than those with controlled hypertension (51.5 vs. $17 \%)^{[12]}$.

Hypertension has been documented as a risk factor in studies from Jordan ${ }^{[43]}$, Oman ${ }^{[17]}$, and also by a longitudinal UK prospective diabetes study group ${ }^{[44]}$.

While in contrast, some studies were not able to find any significant role of hypertension in the development of $\mathrm{DR}^{[45-47]}$.

In the current study, cholesterol level and BCVA had statistically significant predictive ability for macular thickness.

On the other hand, Ahmed et al. (2016) reported that by univariable analysis, the rate of retinopathy was significantly associated with older age group, younger age at onset, longer duration of disease, poorly controlled blood sugar, the presence of hypertension (receiving drug treatment), insulin use, and the presence of multiple complications ${ }^{[8]}$.

Our findings also disagreed with the study in Oman in which similarly aged patients, poor control of diabetes (with HbAlc $\geq 9$ ), high systolic blood pressure and complications were documented as insignificant after multiple regression analysis ${ }^{[17]}$.

The difference could be attributed to many factors including different sample size, different patients' characteristics, different techniques of assessment and the high prevalence of metabolic syndrome and dyslipidemia (Even if not diagnosed) among our study population. 
The limitation of our study is that a predictive inference cannot be drawn from our observational data since a more extensive study is required. Moreover, the relatively small sample size (according to the nature of the study) could decrease the power of the obtained results.

\section{Conclusion:}

Our finding clearly demonstrates that the retina of diabetic patient provides a summary measure of lifetime exposure to the effects of hyperglycaemia.

We supported the idea that good control of diabetes will not prevent the occurrence of DR but it can delay the onset, progression and complication of diabetic retinopathy.

Small investments in prevention, awareness and care can dramatically improve the quality of life of patients with long-standing diabetes.

The treating physicians should also be reminded to refer all diabetics to ophthalmologists for examination. Much awareness and attention to this complication is therefore needed in Egypt.

\section{Conflict of Interest}

Authors declare no conflicts of interest.

\section{Corresponding author}

Correspondence to: Amira Ahmad Kashwa

Email: amirakashwa@gmail.com

\section{Affiliations}

Amira Ahmad Kashwa MBBCH, Mansoura University Department of Ophthalmology, Faculty of Medicine, Mansoura University, Egypt.

\section{Ethics declarations}

\section{Conflict of interest}

Amira Kashwa, Amr M Abdelkader, Hossam Eldin Abouelkheir, Hamza Ahmad, all authors have no conflicts of interest that are directly relevant to the content of this review.

Funding: No sources of funding were used to conduct this review.

Reviewer disclosures: No relevant financial or other relationships to disclose.

Declaration of interest: No financial affiliations or financial involvement with any organization or entity with a financial competing with the subject matter or materials discussed in the review.

\section{References}

1. Walinjkar RS, Khadse S, Kumar S, Bawankule S, Acharya S. Platelet indices as a predictor of microvascular complications in type 2 diabetes. Indian journal of endocrinology and metabolism. 2019;23(2):206.

2. Animaw W, Seyoum Y. Increasing prevalence of diabetes mellitus in a developing country and its related factors. PloS one. 2017;12(11):e0187670.

3. Kumari N, Karmakar A, Ganesan SK. Targeting epigenetic modifications as a potential therapeutic option for diabetic retinopathy. Journal of cellular physiology. 2020;235(3):1933-1947.

4. Semeraro F, Morescalchi F, Cancarini A, Russo A, Rezzola S, Costagliola C. Diabetic retinopathy, a vascular and inflammatory disease: therapeutic implications. Diabetes \& metabolism. 2019;45(6):517527.

5. Tang FY, Ng DS, Lam A, Luk F, Wong R, Chan C, et al. Determinants of quantitative optical coherence tomography angiography metrics in patients with diabetes. Scientific reports. 2017;7(1):1-10.

6. Al-Maskari F, El-Sadig M. Prevalence of diabetic retinopathy in the United Arab Emirates: a crosssectional survey. BMC ophthalmology. 2007;7(1):1-8.

7. Nwanyanwu KH, Talwar N, Gardner TW, Wrobel JS, Herman WH, Stein JD. Predicting development of proliferative diabetic retinopathy. Diabetes care. 2013;36(6):1562-1568.

8. Ahmed RA, Khalil SN, Al-Qahtani MA. Diabetic retinopathy and the associated risk factors in diabetes type 2 patients in Abha, Saudi Arabia. Journal of family \& community medicine. 2016;23(1):18.

9. Rathod KS, Hamshere SM, Jones DA, Mathur A. Intravascular ultrasound versus optical coherence tomography for coronary artery imaging-apples and oranges? Interventional Cardiology Review. 2015;10(1):8.

10. Sandhu HS, Eltanboly A, Shalaby A, Keynton RS, Schaal S, El-Baz A. Automated diagnosis and grading of diabetic retinopathy using optical coherence 
tomography. Investigative ophthalmology \& visual science. 2018;59(7):3155-3160.

11. Abdeen GM, Radwan G, Elagouz M, Farouk MM. Optical Coherence Tomography Findings of Diabetic Maculopathy in Egypt. Ophthalmology Research: An International Journal. 2018:1-7.

12. Macky TA, Khater N, Al-Zamil MA, El Fishawy H, Soliman MM. Epidemiology of diabetic retinopathy in Egypt: a hospital-based study. Ophthalmic research. 2011;45(2):73-78.

13. Herman W, Aubert R, Engelgau M, Thompson T, Ali M, Sous E, et al. Diabetes mellitus in Egypt: glycaemic control and microvascular and neuropathic complications. Diabetic Medicine. 1998;15(12):10451051.

14. El-Asrar AMA, Al-Rubeaan KA, Al-Amro SA, Kangave D, Moharram OA. Risk factors for diabetic retinopathy among Saudi diabetics. International ophthalmology. 1998;22(3):155-161.

15. Bamashmus MA, Gunaid AA, Khandekar R. Regular visits to a diabetes clinic were associated with lower magnitude of visual disability and diabetic retinopathy - a hospital-based historical cohort study in yemen. Diabetes technology \& therapeutics. 2009;11(1):45-50.

16. Waked N, Nacouzi R, Haddad N, Zaini R. Epidemiology of diabetic retinopathy in Lebanon. Journal francais d'ophtalmologie. 2006;29(3):289-295.

17. El Haddad OA, Saad MK. Prevalence and risk factors for diabetic retinopathy among Omani diabetics. British journal of ophthalmology. 1998;82(8):901-906.

18. Zheng Y, He M, Congdon N. The worldwide epidemic of diabetic retinopathy. Indian journal of ophthalmology. 2012;60(5):428.

19. Saadi H, Carruthers SG, Nagelkerke N, Al-Maskari F, Afandi B, Reed R, et al. Prevalence of diabetes mellitus and its complications in a population-based sample in $\mathrm{Al}$ Ain, United Arab Emirates. Diabetes research and clinical practice. 2007;78(3):369-377.

20. Khandekar R, Al Lawatii J, Mohammed A, Al Raisi A. Diabetic retinopathy in Oman: a hospital based study.
British journal of ophthalmology. 2003;87(9):10611064.

21. Al-Adsani A. Risk factors for diabetic retinopathy in Kuwaiti type 2 diabetic patients. Saudi medical journal. 2007;28(4):579-583.

22. El-Bab MF, Shawky N, Al-Sisi A, Akhtar M. Retinopathy and risk factors in diabetic patients from Al-Madinah Al-Munawarah in the Kingdom of Saudi Arabia. Clinical Ophthalmology (Auckland, NZ). 2012;6:269.

23. Bloomgarden ZT. Screening for and managing diabetic retinopathy: current approaches. American Journal of health-system pharmacy. 2007;64(17_Supplement_12):S8-S14.

24. Zhang X, Saaddine JB, Chou C-F, Cotch MF, Cheng YJ, Geiss LS, et al. Prevalence of diabetic retinopathy in the United States, 2005-2008. Jama. 2010;304(6):649-656.

25. Wild S, Roglic G, Green A, Sicree R, King H. Global prevalence of diabetes: estimates for the year 2000 and projections for 2030. Diabetes care. 2004;27(5):10471053.

26. Chen M-S, Kao C-S, Chang C-J, Wu T-J, Fu C-C, Chen C-J, et al. Prevalence and risk factors of diabetic retinopathy among noninsulin-dependent diabetic subjects. American journal of ophthalmology. 1992;114(6):723-730.

27. Jerneld B, Algvere P. Relationship of duration and onset of diabetes to prevalence of diabetic retinopathy. American journal of ophthalmology. 1986;102(4):431437.

28. Raman R, Rani PK, Rachepalle SR, Gnanamoorthy P, Uthra S, Kumaramanickavel G, et al. Prevalence of diabetic retinopathy in India: Sankara Nethralaya diabetic retinopathy epidemiology and molecular genetics study report 2. Ophthalmology. 2009;116(2):311-318.

29. Khan AR, Wiseberg JA, Lateef ZAA, Khan SA. Prevalence and determinants of diabetic retinopathy in Al Hasa region of Saudi Arabia: primary health care centre based cross-sectional survey, 2007-2009. Middle East African journal of ophthalmology. 2010;17(3):257. 
30. Al Ghamdi AH, Rabiu M, Hajar S, Yorston D, Kuper H, Polack S. Rapid assessment of avoidable blindness and diabetic retinopathy in Taif, Saudi Arabia. British Journal of Ophthalmology. 2012;96(9):1168-1172.

31. Stratton I, Kohner E, Aldington S, Turner R, Holman R, Manley S, et al. UKPDS 50: risk factors for incidence and progression of retinopathy in Type II diabetes over 6 years from diagnosis. Diabetologia. 2001;44:156-163.

32. Jones CD, Greenwood RH, Misra A, Bachmann MO. Incidence and progression of diabetic retinopathy during 17 years of a population-based screening program in England. Diabetes care. 2012;35(3):592-596.

33. Lee R, Wong TY, Sabanayagam C. Epidemiology of diabetic retinopathy, diabetic macular edema and related vision loss. Eye and vision. 2015;2(1):1-25.

34. Sjølie AK, Klein R, Porta M, Orchard T, Fuller J, Parving HH, et al. Effect of candesartan on progression and regression of retinopathy in type 2 diabetes (DIRECT-Protect 2): a randomised placebo-controlled trial. The Lancet. 2008;372(9647):1385-1393.

35. Ismail-Beigi F, Craven T, Banerji MA, Basile J, Calles $\mathrm{J}$, Cohen RM, et al. Effect of intensive treatment of hyperglycaemia on microvascular outcomes in type 2 diabetes: an analysis of the ACCORD randomised trial. The Lancet. 2010;376(9739):419-430.

36. Network DRCR. Relationship between optical coherence tomography-measured central retinal thickness and visual acuity in diabetic macular edema. Ophthalmology. 2007;114(3):525-536.

37. Park Y-H, Shin JA, Han J-H, Park Y-M, Yim HW. The association between chronic kidney disease and diabetic retinopathy: the Korea National Health and Nutrition Examination Survey 2008-2010. PLoS One. 2015;10(4):e0125338.

38. Xu J, Xu L, Wang YX, You QS, Jonas JB, Wei WB. Ten-year cumulative incidence of diabetic retinopathy. The Beijing Eye Study 2001/2011. PLoS One. 2014;9(10):e111320.
39. Das R, Kerr R, Chakravarthy U, Hogg RE. Dyslipidemia and diabetic macular edema: a systematic review and meta-analysis. Ophthalmology. 2015;122:1820-1827.

40. Zheng Y, Lamoureux EL, Lavanya R, Wu R, Ikram MK, Wang JJ, et al. Prevalence and risk factors of diabetic retinopathy in migrant Indians in an urbanized society in Asia: the Singapore Indian eye study. Ophthalmology. 2012;119(10):2119-2124.

41. Jee D, Lee WK, Kang S. Prevalence and risk factors for diabetic retinopathy: the Korea National Health and Nutrition Examination Survey 2008-2011. Investigative ophthalmology \& visual science. 2013;54(10):68276833.

42. Keech AC, Mitchell P, Summanen P, O'Day J, Davis TM, Moffitt M, et al. Effect of fenofibrate on the need for laser treatment for diabetic retinopathy (FIELD study): a randomised controlled trial. The Lancet. 2007;370(9600):1687-1697.

43. Al-Bdour MD, Al-Till MI, Samra KMA. Risk factors for diabetic retinopathy among Jordanian diabetics. Middle East African journal of ophthalmology. 2008;15(2):77.

44. UK Prospective Diabetes Study Group. Tight blood pressure control and risk of macrovascular and microvascular complications in type 2 diabetes: UKPDS 38. Bmj. 1998;317(7160):703-713.

45. Rema M, Ponnaiya M, Mohan V. Prevalence of retinopathy in non insulin dependent diabetes mellitus at a diabetes centre in southern India. Diabetes research and clinical practice. 1996;34(1):29-36.

46. Maghbooli Z, Pasalar P, Keshtkar A, Farzadfar F, Larijani B. Predictive factors of diabetic complications: a possible link between family history of diabetes and diabetic retinopathy. Journal of diabetes \& metabolic disorders. 2014;13(1):1-5.

47. van Leiden HA, Dekker JM, Moll AC, Nijpels G, Heine RJ, Bouter LM, et al. Risk factors for incident retinopathy in a diabetic and nondiabetic population: the Hoorn study. Archives of ophthalmology. 2003;121(2):245-251. 